\title{
Comparison of two dose and three dose human papillomavirus vaccine schedules: cost effectiveness analysis based on transmission model
}

\author{
OPEN ACCESS
}

\author{
Mark Jit mathematical modeller and health economist ${ }^{12}$, Marc Brisson associate professor of \\ mathematical epidemiology and health economics ${ }^{345}$, Jean-François Laprise mathematical modeller ${ }^{3}$, \\ Yoon Hong Choi mathematical modeller ${ }^{16}$
}

\begin{abstract}
${ }^{1}$ Modelling and Economics Unit, Public Health England, London NW9 5EQ, UK; ${ }^{2}$ Department of Infectious Disease Epidemiology, London School of Hygiene and Tropical Medicine, London; ${ }^{3}$ SP-POS, Centre de recherche du CHU de Québec, 1050 Chemin Sainte-Foy, Québec, Canada; ${ }^{4}$ Department of Infectious Disease Epidemiology, Imperial College, London; ${ }^{5}$ Département de médecine sociale et préventive, Université Laval, Québec; ${ }^{6}$ Immunisation, Hepatitis and Blood Safety Department, Public Health England, London
\end{abstract}

\begin{abstract}
Objective To investigate the incremental cost effectiveness of two dose human papillomavirus vaccination and of additionally giving a third dose.

Design Cost effectiveness study based on a transmission dynamic model of human papillomavirus vaccination. Two dose schedules for bivalent or quadrivalent human papillomavirus vaccines were assumed to provide 10,20 , or 30 years' vaccine type protection and cross protection or lifelong vaccine type protection without cross protection. Three dose schedules were assumed to give lifelong vaccine type and cross protection.
\end{abstract}

Setting United Kingdom.

Population Males and females aged 12-74 years.

Interventions No, two, or three doses of human papillomavirus vaccine given routinely to 12 year old girls, with an initial catch-up campaign to 18 years.

Main outcome measure Costs (from the healthcare provider's perspective), health related utilities, and incremental cost effectiveness ratios.

Results Giving at least two doses of vaccine seems to be highly cost effective across the entire range of scenarios considered at the quadrivalent vaccine list price of $£ 86.50$ (€109.23; \$136.00) per dose. If two doses give only 10 years' protection but adding a third dose extends this to lifetime protection, then the third dose also seems to be cost effective at £86.50 per dose (median incremental cost effectiveness ratio $£ 17000$, interquartile range $£ 11700-£ 25800$ ). If two doses protect for more than 20 years, then the third dose will have to be priced substantially lower (median threshold price $£ 31$, interquartile range $£ 28-£ 35$ ) to be cost effective. Results are similar for a bivalent vaccine priced at $£ 80.50$ per dose and when the same scenarios are explored by parameterising a Canadian model (HPV-ADVISE) with economic data from the United Kingdom.

Conclusions Two dose human papillomavirus vaccine schedules are likely to be the most cost effective option provided protection lasts for at least 20 years. As the precise duration of two dose schedules may not be known for decades, cohorts given two doses should be closely monitored.

\section{Introduction}

Persistent infection with a high risk human papillomavirus type is necessary for cervical cancer. Two human papillomavirus vaccines are available - a bivalent vaccine with antigens for human papillomavirus 16 and 18 associated with $70-80 \%$ of cervical cancers globally (Cervarix) and a quadrivalent vaccine that additionally contains antigens for human papillomavirus 6 and 11 associated with most cases of anogenital warts (Gardasil). Female participants receiving three doses of either vaccine in trials were protected against persistent infection and pre-cancerous lesions associated with human papillomavirus 16 and 18. Universal human papillomavirus vaccination of girls before their sexual debut has been found to be cost effective in both developed and developing countries. ${ }^{12}$ However, the high cost of purchase and delivery of vaccine has been a barrier to more widespread implementation-for example, in developing countries and in demographic groups beyond young adolescent girls. ${ }^{3}$ Furthermore, the need to deliver three doses of a vaccine to a non-traditional age group can be challenging. 
Recent clinical trial and post-introduction data suggest that two doses of human papillomavirus vaccine may be sufficient protection for girls aged 9-14 years when administered in a prime-boost schedule with at least six months between doses to allow affinity maturation of memory B cells elicited by the first dose. ${ }^{4}$ Efficacy against persistent human papillomavirus $16 / 18$ infection in vaccinees who received two bivalent vaccine doses was non-inferior for at least three years after vaccination compared with those who received three doses in a post-hoc non-randomised study in Costa Rica. ${ }^{5}$ In addition, immune responses in 9-14 year old girls after two bivalent vaccine doses six months apart were non-inferior for at least four years to responses in 15-25 year old female patients receiving three doses ${ }^{6}$ and similar results were seen for the quadrivalent vaccine. $^{7}$ Although the Costa Rican study found no evidence of cross protection, an exploratory analysis of a bivalent vaccine trial suggested that antibody responses for human papillomavirus 31 and 45 in 9-14 year old girls after a two dose schedule were comparable to those for three doses in 15-25 year olds. ${ }^{6}$ As three doses have been shown to protect against persistent infection for almost a decade with no evidence of waning, ${ }^{89}$ two doses will probably also provide long lasting protection. The European Medicines Agency has granted marketing authorisation for two dose schedules of both vaccines for girls below 15 years on the basis of these data. ${ }^{10}$

Although two doses are likely to protect vaccinees for more than a decade, whether protection (and potential cross protection) will be as long lasting or broad as it is for three doses is uncertain, as immunogenicity is inferior to that for three doses in equivalent aged girls (rather than in 15-25 year olds). Consideration of two dose schedules as an alternative to three dose schedules relies on comparing the potential benefits of the two dose schedule (cost savings, logistical simplicity, and potentially higher uptake) with its risks (potentially lower protection). As the precise duration and extent of two dose protection is not yet known for certain, this requires consideration of the range of possibilities that are consistent with the available data. A cost effectiveness framework allows an assessment of whether the economic benefits of two dose schedules compensate for its associated risks and uncertainties.

Several jurisdictions have recently switched from three dose to two dose vaccination schedules for girls aged 9-14 years on the basis of such risk-benefit considerations, including Switzerland, the Netherlands, Mexico, the United Kingdom, and Quebec. In two jurisdictions (United Kingdom and Quebec), the decision was explicitly made after consideration of cost effectiveness modelling. ${ }^{11}{ }^{12}$ The World Health Organization's Strategic Advisory Group of Experts on Immunization (SAGE) also recommended a two dose schedule for girls aged 9-14 years after reviewing evidence including cost effectiveness modelling. ${ }^{4}$

Here we present a cost effectiveness analysis of two versus three dose human papillomavirus vaccination; this analysis was used to inform the decisions in the United Kingdom and Quebec, as well as by SAGE to recommend a two dose schedule. The work builds on previous modelling showing that two dose vaccination in England and Canada can provide most of the reduction in cervical cancer associated with three dose vaccination. ${ }^{13}$ In this analysis, we extend the previous model to consider the full range of human papillomavirus related diseases, including other sites of cancer, anogenital warts, and recurrent respiratory papillomatosis. This allows us to examine the cost effectiveness of vaccination, particularly when the duration of protection of two dose vaccination is assumed to be only 10 years, and hence the difference in reduction of cervical cancer between the two schedules is greatest (assuming no booster dose is administered at the point of waning).

\section{Methods}

We previously constructed a model of human papillomavirus transmission, vaccination, and screening to explore the population level effect and cost effectiveness of various options for three dose human papillomavirus vaccination of females in the United Kingdom. ${ }^{14}{ }^{15}$ More recently, this model was adapted to incorporate a two dose option and to explore the differential epidemiological impact of two and three dose schedules on incidence of cervical cancer. ${ }^{13}$

Women were assumed to undergo cytological screening, with women aged 25-49 years screened every three years on average and women aged 50-64 years screened every five years, as recommended by the 2010 National Cervical Screening Programme guidelines. ${ }^{16}$ Uptake, sensitivity, and specificity of screening and efficacy of treatment were based on values used in our previous analyses. ${ }^{17}$ Use of human papillomavirus DNA testing either as triage or as the primary screen was not incorporated.

Modelling and data analysis were conducted in $\mathrm{C}++$ and $\mathrm{R}$. For this analysis, we extended our recent exploration of cervical cancer outcomes to capture the full range of human papillomavirus related outcomes (vulvar, vaginal, anal, penile, and oropharyngeal cancer; anogenital warts; and recurrent respiratory papillomatosis). ${ }^{13} \mathrm{We}$ assumed the quadrivalent vaccine to provide some protection against all these outcomes and the bivalent vaccine to protect only against vulvar, vaginal, anal, penile, and oropharyngeal cancer. Cross protection against non-vaccine types was assumed at the level reported in a recent meta-analysis ${ }^{18}$ with better cross protection from the bivalent vaccine than the quadrivalent one.

We then used all outcomes to compare healthcare costs (from a healthcare provider's perspective) and health related utilities (measured in quality adjusted life years or QALYs) for different schedules for a time horizon of 100 years. We used this to estimate the incremental cost effectiveness of two dose schedules (compared with no vaccination) and three dose schedules (compared with two dose schedules). We assumed vaccination to be given annually to 12 year old girls at $80 \%$ coverage, with a catch-up campaign in the first year to age 18 . For the two dose schedules, we assumed girls up to age 14 to be given two doses and girls older than this to be given three doses. We assumed no booster doses at any age. Economic parameters and the detailed model structure used for the cost effectiveness analysis have been previously described.$^{15} \mathrm{We}$ did probabilistic sensitivity analysis by Latin hypercube sampling from the joint distribution of the economic parameters together with 100 outcome scenarios from the vaccine impact model that best fits pre-vaccination epidemiological data.

Clearly, if two doses of human papillomavirus vaccine are equivalent in every way to three doses for disease prevention, then giving the third dose has no health or economic advantage. To explore situations in which this is not the case, we assumed that three dose schedules gave lifelong protection, with full protection against cervical infection and disease due to human papillomavirus $16 / 18$. We estimated efficacy against human papillomavirus 31/33/45/52/58 from a meta-analysis of quadrivalent vaccine trials. ${ }^{18} \mathrm{We}$ then assumed two dose schedules either to provide reduced duration of protection $(10$, 20 , or 30 years) or to lose efficacy against human papillomavirus $31 / 33 / 45 / 52 / 58$. We assumed girls to be given quadrivalent human papillomavirus vaccine. We then explored the predicted 
impact of two dose schedules in terms of reduction in cervical cancer.

As the cost of vaccine procurement is unknown, we assumed a single dose of vaccine to cost $£ 80.50$ ( $€ 101.65 ; \$ 126.57)$ or $£ 86.50$ (the current list price for bivalent and quadrivalent vaccines in the United Kingdom ${ }^{19}$ ), with $£ 9.33$ administration cost per dose. UK tender prices are unknown but are likely to be substantially lower, so we also did a threshold analysis to find the maximum price at which two dose vaccination and three dose vaccination become cost effective. We used a threshold of $£ 30000$ for an intervention to be cost effective, which is the upper limit of the threshold used by the National Institute for Health and Care Excellence (NICE) ${ }^{20}$ All other costs came from our previous work, but inflated to 2012-13 prices. ${ }^{14} 15$

Costs and benefits were discounted at $3.5 \%$ per annum, as recommended in the NICE reference case..$^{20}$ As NICE guidelines also suggest that a discount rate of $1.5 \%$ per annum can be considered for public health interventions or interventions that have long term health benefits, we also did sensitivity analyses using this rate.

We also explored the same scenarios about two and three dose vaccination by using HPV-ADVISE, an individual based, transmission dynamic model of human papillomavirus vaccination fitted to Canadian data. ${ }^{21}{ }^{22}$ We retained the original model structure and parameters governing infection transmission and disease natural history; however, we adjusted parameters governing cancer mortality, incidence and costs of anogenital warts, and some utility losses associated with human papillomavirus related disease to reflect UK data (see table $1 \Downarrow$ and supplementary tables A and B for details).

\section{Results}

A two dose human papillomavirus vaccination programme is expected to substantially decrease the incidence of human papillomavirus related cancers and anogenital warts (fig $1 \Downarrow$ ). If the duration of protection of a two dose schedule is at least 20 years, then the additional benefit of the third dose is minimal, regardless of whether the vaccine provides cross protection. A two dose schedule giving 20 years' protection may be sufficient to eliminate human papillomavirus $6 / 11$ associated anogenital warts, so a third dose may have little or no long term benefit in terms of protection against warts. If the duration of protection with a two dose schedule is only 10 years, then the benefit of a third dose in terms of reduction in all the human papillomavirus related endpoints examined is greater, although it is still much smaller than the benefit of the first two doses.

Similarly, the healthcare costs and QALYs saved by adding a third dose are minimal when a two dose schedule gives at least 20 years' protection but substantial if it gives only 10 years' protection (fig $2 \Downarrow$ ). Adding a third dose to a two dose schedule with 10 years' protection will in particular save costs associated with cervical screening and warts.

Giving the first two doses of human papillomavirus vaccine is clearly cost effective at a threshold of $£ 20000$ to $£ 30000$ per QALY gained, regardless of the duration of protection (within the range examined), existence of cross protection, and discount rate. At $3.5 \%$ discounting, giving the third dose is highly unlikely to be cost effective if the first two doses give at least 20 years' protection. However, if the first two doses only give 10 years' protection, and the third dose can extend this to lifetime protection, then the third dose becomes cost effective, with a median incremental cost effectiveness ratio of $£ 17000$ (interquartile range $£ 11$ 700-£25 800) per QALY gained (fig
$3 \Downarrow$ (left, solid lines) and supplementary table D). In terms of threshold costs, the first two doses would be clearly cost effective even if the vaccine costs $£ 300$ per dose, well above the list price of $£ 86.50$ per dose. The third dose is cost effective if it extends the duration of protection from 10 years to lifelong and costs less than about $£ 147$ ( $£ 108-188$ ) per dose (including administration costs). However, if two doses give longer than 10 years' protection, then in all the scenarios explored in this model the threshold price per dose for the third dose remains below $£ 31$ ( $£ 28-35$ ), and may be substantially less depending on exact assumptions about duration of protection and cross protection (fig $4 \Downarrow$ (left, solid lines) and supplementary table D). The total costs of vaccination are shown in supplementary table C.

Overall conclusions are also similar if a bivalent vaccine is used instead of a quadrivalent vaccine (fig $5 \Downarrow$; see supplementary figures A and B for detailed breakdowns). Giving a third dose is still cost effective only if it extends the duration of protection by vaccine from 10 years to lifelong. The assumed superior cross protection and lower vaccine cost of the bivalent vaccine partially compensate for the loss of protection against warts and recurrent respiratory papillomatosis. If a discount rate of $1.5 \%$ per annum instead of $3.5 \%$ per annum is used, then the third dose becomes slightly more cost effective, because outcomes that occur further in the future and are prevented by having a longer duration of protection are valued more (figures $3 \Downarrow$ and $4 \Downarrow$, right, solid lines). However, the overall conclusions are unchanged.

HPV-ADVISE gives qualitatively similar results to the UK model but is slightly more favourable to giving the third dose (figures $3 \Downarrow$ and $4 \Downarrow$ (left, dashed lines) and supplementary table E). If two dose protection lasts longer than 20 years, then the incremental cost effectiveness ratio for giving the third dose also lies above $£ 30000$ per QALY gained at the list price of $£ 86.50$ per dose. The threshold cost for the third dose of quadrivalent vaccine to be cost effective is predicted to be $£ 26$ (£14-43), £2 (£0-7), and £26 (£15-29), respectively (including administration costs), for the scenarios in which the first two doses give 20 years' duration, 30 years' duration, and lifetime duration but no cross protection. If the first two doses give only 10 years' protection and the third dose gives lifetime protection, then, like the UK model, HPV-ADVISE suggests that the third dose is very likely to be cost effective. In this scenario, the incremental cost effectiveness ratio of the third dose is only $£ 9700$ ( $£ 8900-£ 11000)$ at the vaccine list price. Results for the bivalent vaccine are similar, with the third dose again being cost effective only if it extends protection from 10 years to lifetime.

\section{Discussion}

If the quadrivalent human papillomavirus vaccine costs $£ 86.50$ per dose or less to procure and distribute, then giving 12 year old girls at least two doses of vaccine is clearly cost effective. However, the cost effectiveness of giving the third dose of vaccine is highly dependent on how well the first two doses protect. If two doses protect for only 10 years but adding a third dose extends this to lifetime protection, then the third dose may be cost effective even if giving a dose of vaccine costs $£ 86.50$ or more. Conversely, if two doses protect for 20 years or more, or if they give lifetime protection against vaccine type disease but without cross protection, then the third dose has to be substantially cheaper than its list price to be cost effective. Overall conclusions are the same for the bivalent vaccine, although the cost effectiveness of two versus three dose 
schedules differs slightly owing to differing cross protection and protection against warts. Also, evidence about the efficacy and immunogenicity of two doses of each of the two vaccines is not equivalent. For example, no clinical efficacy data using two doses of the quadrivalent vaccine is available.

The same conclusions hold when equivalent scenarios are explored using HPV-ADVISE fitted to Canadian data on human papillomavirus epidemiology and sexual mixing, but using economic parameters from the United Kingdom. HPV-ADVISE is slightly more favourable to three dose vaccination compared with results from the UK model, particularly when two doses of human papillomavirus vaccine provide only 10 years of protection. The reasons for this have been previously explored ${ }^{13}$; they are likely to be differences between self reported rates of change of sexual partner in UK and Canadian women beyond the 20-25 year old age group (when they would no longer be protected by a two dose vaccine giving only 10 years' protection), as well as the way this is represented in the two models.

\section{Strengths and limitations}

Both the UK model and HPV-ADVISE are transmission dynamic models that take into account indirect (herd) vaccine protection due to reduced transmission of human papillomavirus. Herd protection is likely to be a substantial contributor to the impact of two dose programmes with less than lifetime duration of protection. Models also incorporate the full range of human papillomavirus related outcomes, including cervical cancer (and its precursors), other cancers, and anogenital warts. The consistency of results between the two models despite differences in underlying human papillomavirus epidemiology represented in each of them suggests that the main conclusions are generalisable to other high income countries with similar human papillomavirus epidemiology. However, caution is needed in interpreting the results for resource poor settings owing to differences in sexual behaviour, human papillomavirus epidemiology, cervical screening coverage, and healthcare costs. Similar analyses for these settings are a priority, particularly following the Strategic Advisory Group of Experts on Immunization's global recommendation of two dose schedules.

One limitation of this analysis is that it does not capture historical changes in the human papillomavirus vaccination programme in the United Kingdom over the past six years. This includes the introduction of bivalent human papillomavirus in 2008 with a catch-up campaign staggered over two years, a switch to the quadrivalent vaccine in 2011, and a switch from three dose to two dose vaccination in September 2014. Instead, we considered a more general scenario in which quadrivalent vaccination is introduced with either two or three doses from the start of the programme, and with a catch-up programme up to age 18 years. This allows results to be more readily generalised to other settings where human papillomavirus vaccination may be (or has already been) introduced with a different configuration from the United Kingdom. Furthermore, it is a conservative assumption: assuming that the same schedule (two or three dose) is followed from the start of the programme maximises the difference in impact between the two schedules. Conversely, allowing a few years of three dose vaccination will temporarily reduce any potential loss of effectiveness when switching to two doses because of residual herd protection from older vaccinees who have received three doses.

Our model assumed that cervical screening is cytology based. Several countries (such as the Netherlands and Italy) have moved from cytology based to human papillomavirus DNA based detection methods as their primary cervical screening method. ${ }^{23}$ In the United Kingdom, cytology is still used as the primary test, although human papillomavirus DNA testing as primary has been piloted in several areas. ${ }^{24}$ However, countries that have moved to a primary human papillomavirus DNA testing algorithm have been conservative in the move, in that they have chosen an algorithm such that the lifetime risk of cancer in women screened using primary human papillomavirus DNA testing is predicted to be no greater than it was using the former primary cytology based algorithm. Consequently, any change to human papillomavirus testing can only decrease (or leave unchanged) the benefit of giving a third dose of vaccine, as there will be fewer (or the same number of) cancers to prevent. Hence the overall conclusions about giving a third dose not being cost effective if two doses provide at least 20 years' protection would still hold.

\section{Implications and future research}

In the United Kingdom and many other countries with competitive tendering, tender prices for vaccines can be substantially lower than their list price. If two doses give only 20 years' protection, then the threshold price for the third dose to be cost effective is around $£ 16$ with $3.5 \%$ discounting or $£ 32$ with $1.5 \%$ discounting, which may still be within the realms of possibility for the tender price of human papillomavirus vaccines, particularly as vaccine prices mature in the future. However, if 30 years' protection and cross protection can be expected from two doses, then the third dose will be cost effective only if it can be purchased and delivered for less than $£ 3$ a dose (with $3.5 \%$ discounting), which is below the current administration cost for human papillomavirus vaccines in the United Kingdom. ${ }^{15}$

Protection from two doses is likely to be long lasting, but whether it will be as long lasting or broad as it is for three doses is uncertain. There may be no way to determine a priori whether duration of protection from two doses will exceed 30 years. Consequently, dropping the third dose of human papillomavirus vaccine carries a risk as the outcomes relative to retaining three doses are uncertain. This uncertainty cannot be eliminated by modelling. However, economic modelling allows quantification of the relative value of different strategies in terms of their health and economic benefits given their possible outcomes, so that an informed decision about dosage can be made on the basis of weighing risks and benefits. Results of the model also support close monitoring of cohorts given two doses of human papillomavirus vaccines, with the possibility of booster doses or even increasing the number of doses for adolescents if waning is detected decades in the future.

We thank Peter White for helpful comments. We are indebted to Compute Canada for providing us with the power necessary to run the HPV-ADVISE simulations. We also acknowledge Nicolas Van de Velde and Marie-Claude Boily who helped design HPV-ADVISE. In addition, Nicolas Van de Velde programmed most of the core components of HPV-ADVISE.

Contributors: MJ, YHC, and MB designed the study, developed the UK model, and analysed its results. MB co-led the design of HPV-ADVISE. MB and J-FL developed the economic component of HPV-ADVISE and analysed the results. MJ wrote the manuscript with input from all authors. $\mathrm{MJ}$ is the guarantor.

Funding: This research was part funded by the National Institute for Health Research Health Protection Research Unit (NIHR HPRU) in Immunisation at the London School of Hygiene and Tropical Medicine in partnership with Public Health England. MB is supported by the Canada Research Chairs programme. The views expressed are those 


\section{What is already known on this topic}

Two dose human papillomavirus vaccine schedules are likely to provide long lasting protection against human papillomavirus 16 and 18

The exact duration and breadth of two dose protection compared with three dose protection is uncertain

\section{What this study adds}

If two doses of bivalent or quadrivalent human papillomavirus vaccines give only 10 years' protection but adding a third dose extends this to lifetime protection, then the third dose also seems likely to be cost effective

If two doses provide more than 20 years' protection, then they are likely to be the most cost effective option

of the authors and not necessarily those of the NHS, the NIHR, the Department of Health, or Public Health England. The funders had no role in the study design; in the collection, analysis, and interpretation of data; in the writing of the report; or in the decision to submit the article for publication.

Competing interests: All authors have completed the ICMJE uniform disclosure form at www.icmje.org/coi_disclosure.pdf (available on request from the corresponding author) and declare: no support from any organisation for the submitted work other than that detailed above; in the past three years, MB has consulted once for GlaxoSmithKline (for rotavirus vaccine) and his institution has received unrestricted grants from Merck Frosst (none is ongoing); no other relationships or activities that could appear to have influenced the submitted work.

Ethical approval: Not needed, as the study uses only data that have already been published.

Transparency: MJ affirms that the manuscript is an honest, accurate, and transparent account of the study being reported; that no important aspects of the study have been omitted; and that any discrepancies from the study as planned have been explained.

Data sharing: No additional data available.

1 Brisson M, Van de Velde N, Boily MC. Economic evaluation of human papillomavirus vaccination in developed countries. Public Health Genomics 2009;12:343-51.

2 Fesenfeld M, Hutubessy R, Jit M. Cost-effectiveness of human papillomavirus vaccination in low and middle income countries: a systematic review. Vaccine 2013;31:3786-804.

3 Agosti JM, Goldie SJ. Introducing HPV vaccine in developing countries-key challenges and issues. N Engl J Med 2007;356:1908-10.

4 World Health Organization. Summary of the SAGE April 2014 meeting. 2014. www.who. int/immunization/sage/meetings/2014/april/report_summary_april_2014/en.

5 Kreimer AR, Rodriguez AC, Hildesheim A, Herrero R, Porras C, Schiffman M, et al. Proof-of-principle evaluation of the efficacy of fewer than three doses of a bivalent HPV16/18 vaccine. J Nat/ Cancer Inst 2011;103:1444-51.

6 Romanowski B, Schwarz TF, Ferguson LM, Ferguson M, Peters K, Dionne M, et al. Immune response to the HPV-16/18 AS04-adjuvanted vaccine administered as a 2-dose or 3-dose schedule up to 4 years after vaccination: results from a randomized study. Hum Vaccin Immunother 2014:10:1155-65.

7 Dobson SR, McNeil S, Dionne M, Dawar M, Ogilvie G, Krajden M, et al. Immunogenicity of 2 doses of HPV vaccine in younger adolescents vs 3 doses in young women: a randomized clinical trial. JAMA 2013;309:1793-802.

8 Naud PS, Roteli-Martins CM, De Carvalho NS, Teixeira JC, de Borba PC, Sanchez N, et al. Sustained efficacy, immunogenicity, and safety of the HPV-16/18 AS04-adjuvanted vaccine: final analysis of a long-term follow-up study up to 9.4 years post-vaccination. Hum Vaccin Immunother 2014;10:2147-62.
9 Steben, M. Update on Gardasil(R) (quadrivalent human papillomavirus [HPV] 6/11/16/18 vaccine) clinical trial efficacy results. Presented at EUROGIN 2010, Monte Carlo, Monaco, 17-20 February 2010.

10 Stanley MA, Sudenga SL, Giuliano AR. Alternative dosage schedules with HPV virus-like particle vaccines. Expert Rev Vaccines 2014;13:1027-38

11 Joint Committee on Vaccination and Immunisation. 11/12 February 2014: meeting minutes and agenda. www.gov.uk/government/uploads/system/uploads/attachment_data/file/ 294834/JCVI.zip

12 Comité sur l'immunisation du Québec. La vaccination des pré-adolescents contre les virus du papillome humain (VPH) au Québec : deux ou trois doses? 2013. www.inspq.qc. ca/pdf/publications/1683_VaccinPreAdoVPHQc_2ou3Doses.pdf.

13 Jit M, Choi YH, Laprise JF, Boily MC, Drolet M, Brisson M. Two-dose strategies for human papillomavirus vaccination: how well do they need to protect? Vaccine 2014;32:3237-42.

14 Jit M, Choi YH, Edmunds WJ. Economic evaluation of human papillomavirus vaccination in the United Kingdom. BMJ 2008;337:a769.

15 Jit M, Chapman R, Hughes $\mathrm{O}$, Choi YH. Comparing bivalent and quadrivalent human papillomavirus vaccines: economic evaluation based on transmission model. $B M J$ 2011;343:d5775

16 Luesley D, Leeson S. Colposcopy and programme management: guidelines for the NHS Cervical Screening Programme. Second ed. NHS Cancer Screening Programmes, 2010 (available at www.cancerscreening.nhs.uk/cervical/publications/nhscsp20.pdf). (NHSCSP Publication No 20.)

17 Choi YH, Jit M, Gay N, Cox A, Garnett GP, Edmunds WJ. Transmission dynamic modelling of the impact of human papillomavirus vaccination in the United Kingdom. Vaccine 2010:28:4091-102.

18 Malagon T, Drolet M, Boily MC, Franco EL, Jit M, Brisson J, et al. Cross-protective efficacy of two human papillomavirus vaccines: a systematic review and meta-analysis. Lancet Infect Dis 2012;12:781-9.

19 British Medical Association, Royal Pharmaceutical Society of Great Britain. British national formulary. BMA, RPS, 2013. (No 65.)

20 National Institute for Health and Care Excellence. Guide to the methods of technology appraisal. NICE, 2013

21 Van de Velde N, Boily MC, Drolet M, Franco EL, Mayrand MH, Kliewer EV, et al. Population-level impact of the bivalent, quadrivalent, and nonavalent human papillomavirus vaccines: a model-based analysis. J Natl Cancer Inst 2012;104:17121-23.

22 Brisson M, Laprise JF, Drolet M, Van De Velde N, Franco EL, Kliewer EV, et al. Comparative cost-effectiveness of the quadrivalent and bivalent human papillomavirus vaccines: a transmission dynamic modeling study. Vaccine 2013;31:3863-71.

23 Rossi PG, Vicentini M. New cervical cancer screening guidelines on both sides of the Atlantic. EMJ Oncol 2013;1:80-9.

24 Wise J. Pilot study will assess whether HPV test should replace smears to screen for cervical cancer. BMJ 2012;344:e3744.

Accepted: 24 November 2014

\section{Cite this as: BMJ 2015;350:g7584}

This is an Open Access article distributed in accordance with the Creative Commons Attribution Non Commercial (CC BY-NC 4.0) license, which permits others to distribute, remix, adapt, build upon this work non-commercially, and license their derivative works on different terms, provided the original work is properly cited and the use is non-commercial. See: http://creativecommons.org/licenses/by-nc/4.0/. 


\section{Table}

\section{Table 1 | Cost and quality of life parameters used by UK model and HPV-ADVISE}

Mean (95\% uncertainty interval)

Parameter

UK model

HPV-ADVISE

Treatment costs

Cervical cancer

$£ 16400(4360 \text { to } 43700)^{\star}$

$£ 16200$

Vulvar-vaginal cancer

$£ 14900$ (3770 to 40800$)^{\star}$

$£ 12200$

Anal cance

$£ 14300$ (3300 to 40800$)^{\star}$

$£ 10400$

Oropharyngeal cancer

$£ 16300$ (4270 to 43400$)^{\star}$

$£ 16400$

Penile cancer

$£ 12700$ (3340 to 34200$)^{*}$

$£ 10900$

Anogenital warts

$£ 121(112 \text { to } 130)^{\star}$

$£ 121$

Recurrent respiratory papillomatoses:

\begin{tabular}{lcc}
\hline Juvenile & $£ 32300(1100 \text { to } 178000)^{*}$ & Not modelled \\
\hline Adult & $£ 5300(621 \text { to } 20500)^{*}$ & Not modelled
\end{tabular}

Screening costs

\begin{tabular}{|c|c|c|}
\hline Cytology (liquid based) & $£ 58(30 \text { to } 87)^{*}$ & $£ 58$ \\
\hline Colposcopy & $£ 156(80 \text { to } 232)^{*}$ & $£ 156$ \\
\hline Pre-cancerous lesion treatment & $£ 378(193 \text { to } 562)^{*}$ & $£ 378$ \\
\hline \multicolumn{3}{|l|}{ Vaccine costs (per dose) } \\
\hline Quadrivalent vaccine & $£ 86.50 \dagger$ & $£ 86.50$ \\
\hline Bivalent vaccine & $£ 80.50 \dagger$ & $£ 80.50$ \\
\hline Administration & $£ 9.33 \ddagger$ & $£ 9.33$ \\
\hline \multicolumn{3}{|l|}{ Quality of life weights } \\
\hline Cervical cancer treatment & $0.285(0.25 \text { to } 0.32)^{*}$ & 0.30 (stage I-III) or 0.38 (stage IV) \\
\hline Vulvar-vaginal cancer treatment & $0.32(0.19 \text { to } 0.47)^{*}$ & 0.32 \\
\hline Anal cancer treatment & $0.51(0.28 \text { to } 0.76)^{*}$ & 0.51 \\
\hline Oropharyngeal cancer treatment & $0.25(0.21 \text { to } 0.29)^{*}$ & 0.25 \\
\hline Penile cancer treatment & $0.29(0.22 \text { to } 0.36)^{*}$ & 0.29 \\
\hline Recovery from cancer & $0.0305(0.00682 \text { to } 0.0542)^{*}$ & Not modelled \\
\hline \multicolumn{3}{|l|}{ QALY loss per episode } \\
\hline Positive cytology result & $0.025(0.013 \text { to } 0.037)^{\star}$ & Not modelled \\
\hline Positive CIN1 result & $0.012(0.006 \text { to } 0.018)^{*}$ & 0.006 \\
\hline Positive CIN2 result & $0.007(0.004 \text { to } 0.010)^{*}$ & 0.01 \\
\hline Positive CIN3 result & $0.054(0.027 \text { to } 0.080)^{*}$ & 0.01 \\
\hline Episode of anogenital warts & $0.018(0.007 \text { to } 0.030)^{*}$ & 0.018 \\
\hline Episode of recurrent respiratory papillomatosis & $1.3(0.13 \text { to } 5.3)^{*}$ & Not modelled \\
\hline
\end{tabular}

$\mathrm{CIN}=$ cervical intra-epithelial neoplasia; $\mathrm{QALY}=$ quality adjusted life year.

For UK model, figures show means and $2.5 \%$ to $97.5 \%$ centiles of distributions representing uncertainty in corresponding parameter values; some parameters do not match exactly across two models because they are functions of other parameters that are estimated in different ways (see supplementary table B for actual distributions).

${ }^{*}$ From Jit et al (2011), ${ }^{16}$ inflated to 2012/13 prices.

†From British National Formulary 65 (March 2013).

‡From Jit et al (2011). ${ }^{16}$ 


\section{Figures}
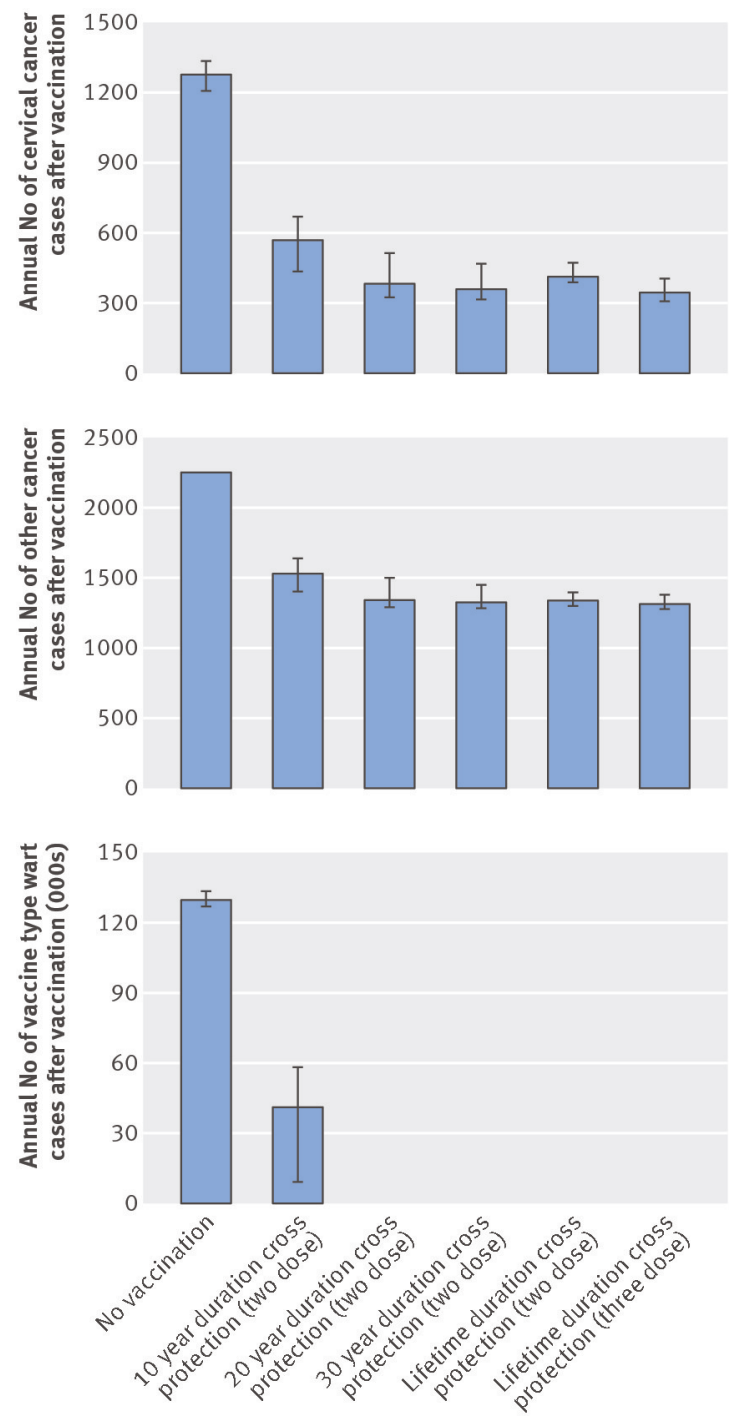

Fig 1 Estimated annual number of cases of cervical cancer, other human papillomavirus related cancers, and vaccine human papillomavirus type warts 100 years after vaccination given no vaccine, two dose quadrivalent vaccination with different characteristics, and three dose vaccination. Results are medians and interquartile ranges of 1000 Latin hypercube samples 

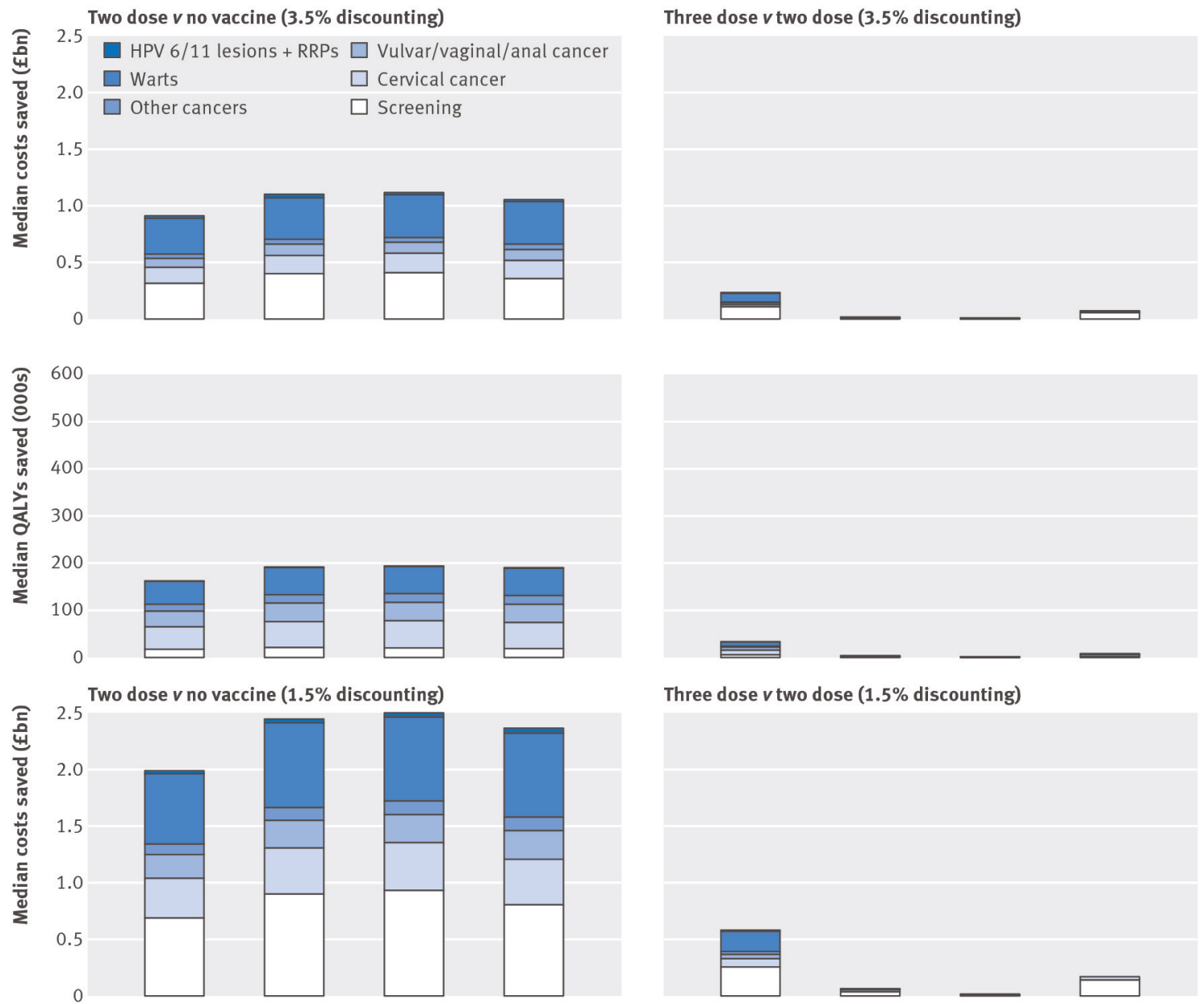

Three dose $v$ two dose (1.5\% discounting)
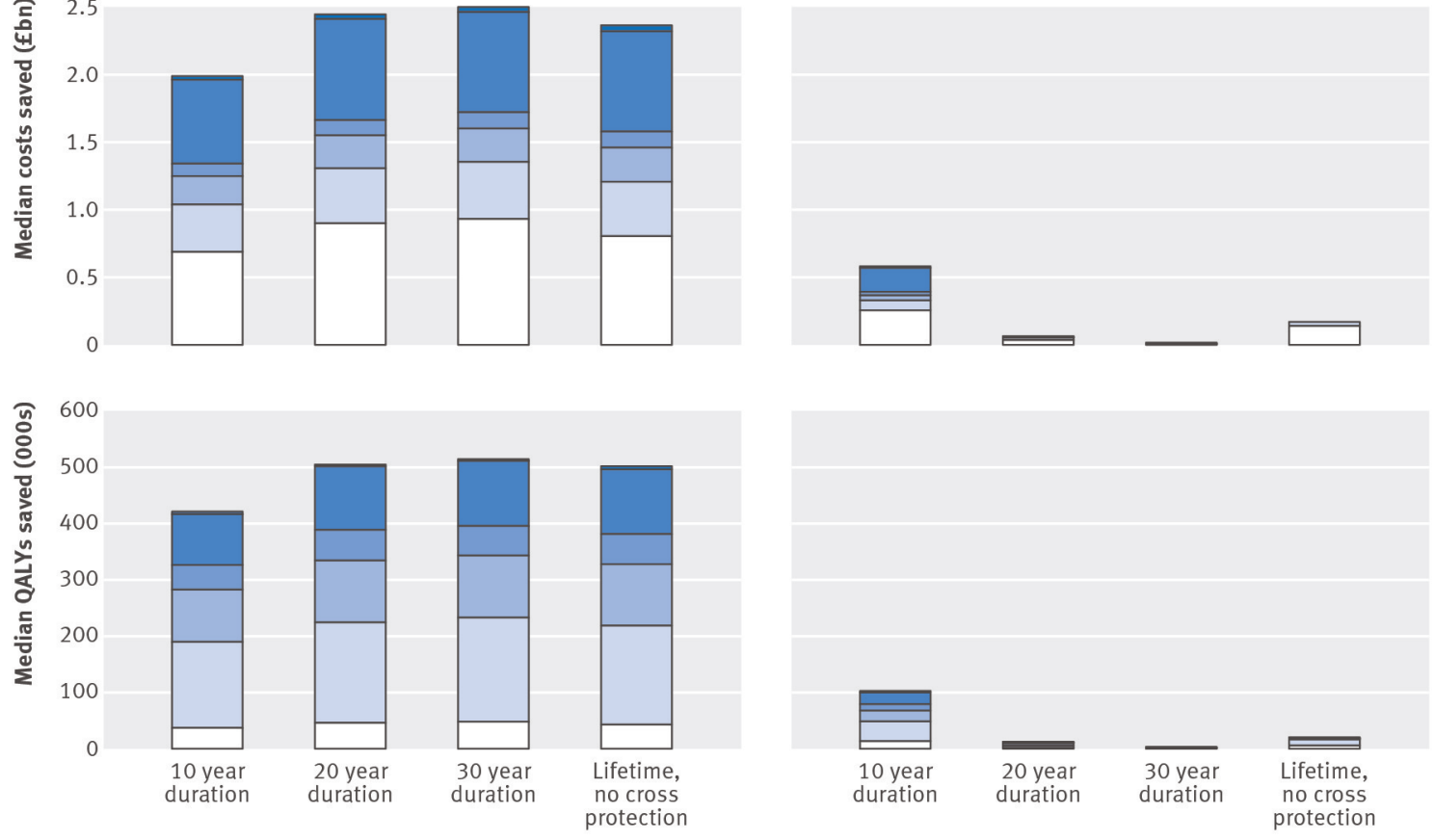

Two dose characteristics

Two dose characteristics

Fig 2 Incremental healthcare costs and quality adjusted life years (QALYs) saved, discounted at 3.5\% or 1.5\% per annum, over 100 years of two dose quadrivalent vaccination (compared with no vaccination) and three dose vaccination (compared with two dose vaccination). Results are medians of 1000 Latin hypercube samples 


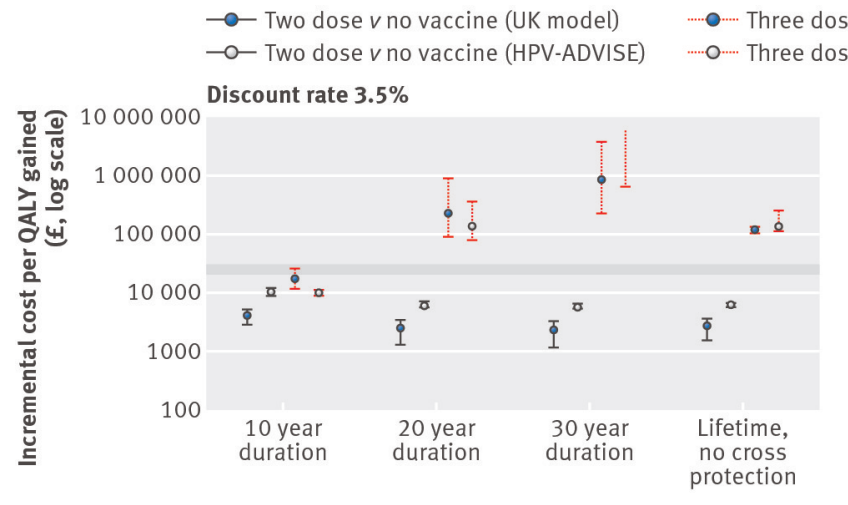

Two dose characteristics
(three dose: lifetime protection and cross protection)

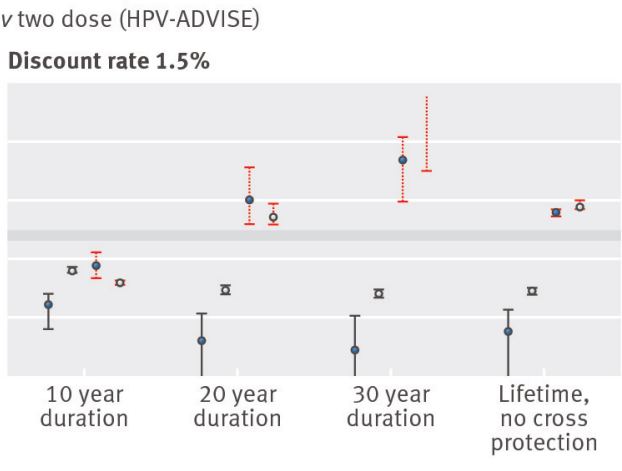

Two dose characteristics

Fig 3 Incremental cost per quality adjusted life year (QALY) gained for two dose quadrivalent vaccination (compared with no vaccination; on logarithmic scale) and three dose quadrivalent vaccination (compared with two dose vaccination). Results are medians and interquartile ranges of 1000 Latin hypercube samples. Results using HPV-ADVISE are shown with dotted bars for comparison. One vaccine dose was assumed to cost $£ 86.50$ for procurement and $£ 9.33$ for administration. Discount rate is 3.5\% (left) or $1.5 \%$ (right) per annum. Shaded area is $£ 20000-£ 30000$ per QALY gained

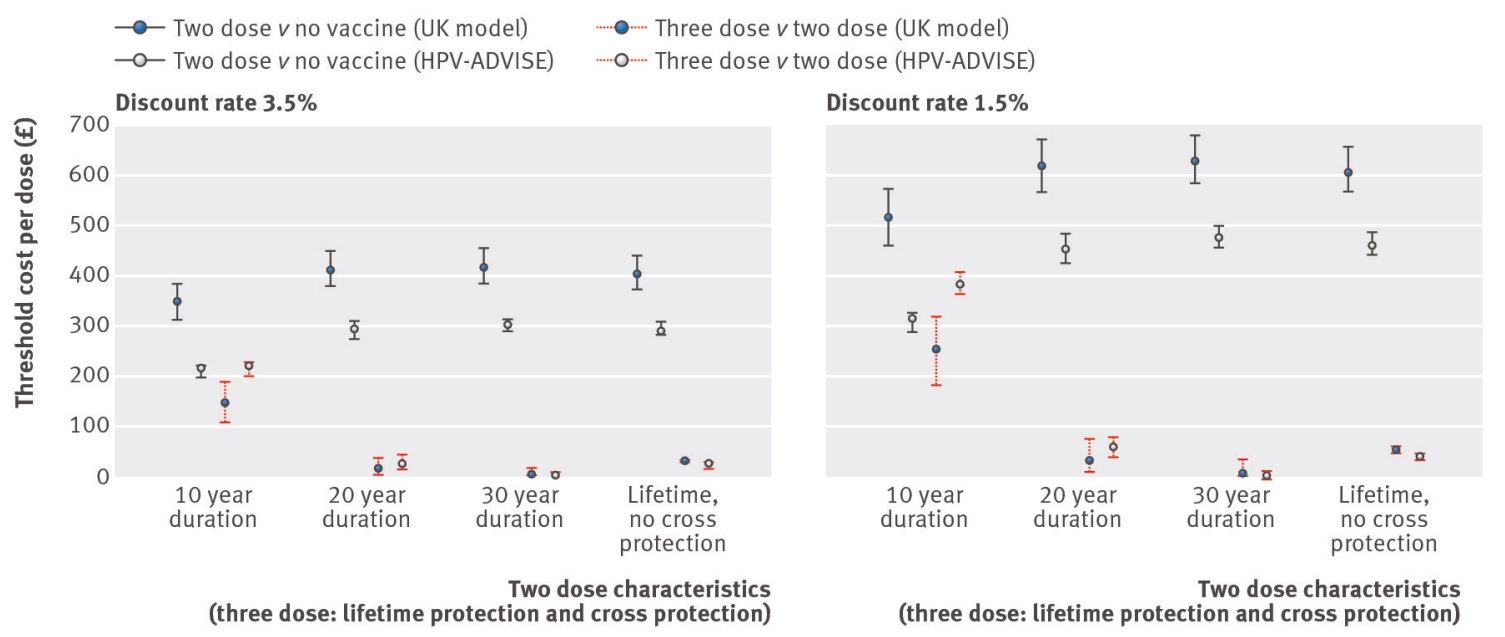

Fig 4 Maximum price per dose (including administration costs) at which two dose quadrivalent vaccination (compared with no vaccination) and three dose quadrivalent vaccination (compared with two dose vaccination) become cost effective, based on threshold of $£ 30000$ for intervention to be cost effective. Results are medians (given in corresponding text) and interquartile ranges of 1000 Latin hypercube samples. Results using HPV-ADVISE are shown with dotted bars for comparison. Discount rate is $3.5 \%$ (left) or $1.5 \%$ (right) per annum 


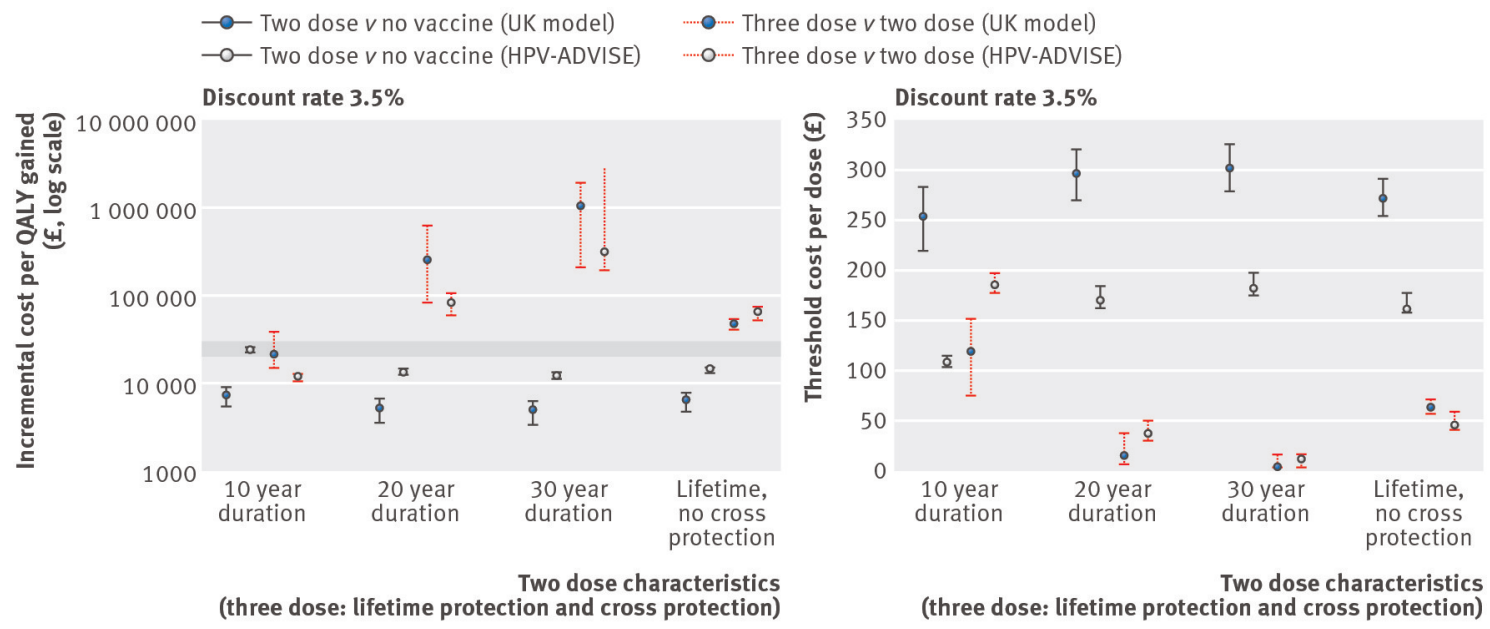

Fig 5 Incremental cost per quality adjusted life year (QALY) and maximum price per dose (including administration costs) at which bivalent vaccination becomes cost effective. One vaccine dose was assumed to cost £86.50 for procurement and $£ 9.33$ for administration. Discount rate is $3.5 \%$. Shaded area is $£ 20000-£ 30000$ per QALY gained 